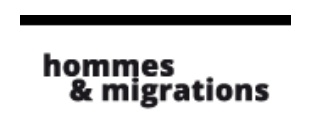

Hommes \& migrations

Revue française de référence sur les dynamiques

migratoires

$1284 \mid 2010$

Migrations et environnement

Kirghizistan

\title{
Kirghizistan. Un scénario où ceux qui partent perdent autant que ceux qui restent
}

\section{Emil Nasritdinov, Mehrigul Ablezova, Jypara Abarikova et Aigoul Abdoubaetova}

Traducteur : Françoise Bouillot

\section{(2) OpenEdition Journals}

\section{Édition électronique}

URL : http://journals.openedition.org/hommesmigrations/1244

DOI : 10.4000/hommesmigrations. 1244

ISSN : 2262-3353

Éditeur

Musée national de l'histoire de l'immigration

Édition imprimée

Date de publication : 1 mars 2010

Pagination : 70-83

ISSN : 1142-852X

Référence électronique

Emil Nasritdinov, Mehrigul Ablezova, Jypara Abarikova et Aigoul Abdoubaetova, « Kirghizistan. Un scénario où ceux qui partent perdent autant que ceux qui restent », Hommes \& migrations [En ligne], 1284 | 2010, mis en ligne le 29 mai 2013, consulté le 19 avril 2019. URL : http://

journals.openedition.org/hommesmigrations/1244; DOI : 10.4000/hommesmigrations.1244 


\section{Kirghizistan Un scénario où ceux qui partent perdent autant que ceux qui restent}

Par Emil Nasritdinov et Mehrigul Ablezova, Centre de recherche sociale, Université américaine d'Asie centrale Jypara Abarikova, Centre régional pour les migrations et les réfugiés et Aigoul Abdoubaetova, Centre de recherche anthropologique "Jorgo", Bichkek

Ancienne république d'URSS, le Kirghizistan est encadré par la Chine à l'est et au sud-est, le Kazakhstan au nord, l'Ouzbékistan à l'ouest et le Tadjikistan au sud-ouest. Extrêmement montagneux, soumis à une forte activité sismique, le pays a longtemps servi de réservoir d'uranium pour le programme nucléaire soviétique. Face aux risques naturels et à la destruction des sols, les Kirghizes, qui tirent une grande part de leurs ressources de l'élevage du bétail, sont forcés de migrer. Mais il est difficile d'échapper à la pollution dans la plus grande décharge nucléaire d'Asie centrale. 
La République kirghize couvre une surface de 1988000 kilomètres carrés (dont $5,3 \%$ de forêts, 4,4 \% de lacs et de rivières, 54,1 \% de terres agricoles, et 36,2 \% classés autres) et $96 \%$ de son territoire est situé à plus de mille mètres au-dessus du niveau de la mer. Environ 30 \% du territoire est habitable, les 70 \% restants étant constitués de hautes montagnes. La chaîne du Pamir-Altay se trouve au sud-ouest. Au nord-est se trouve le Tien Shan et le point culminant de la région, le pic Pobeda (7 $439 \mathrm{~m})$. Ces chaînes de montagnes sont séparées par de profondes vallées et gorges : au nord, les vallées du Chui et de Talas, au sud-ouest la vallée de la Fergana, et au sud la vallée de l'Alay. Le Pamir comme le Tien Shan sont des montagnes assez jeunes et sismiquement très actives. Cette activité sismique et les 7000 mètres de dénivelé entre les hautes et basses altitudes contribuent à de fréquentes catastrophes naturelles, dont la plupart surviennent dans le sud du pays.

Le territoire de la République kirghize est considéré comme le plus dangereux d'Asie centrale sur le plan sismique, et il est sujet à une vingtaine de types de processus naturels dangereux : tremblements de terre, glissements de terrain, inondations, éboulements, avalanches, etc. La fréquence des situations d'urgence majeures en République kirghize se distribue de la façon suivante : inondations, 30 \% ; glissements de terrain, $16 \%$; catastrophes technogéniques, $10 \%$; tremblements de terre, $9 \%$; événements météorologiques, $9 \%$.

Chaque année, les sismologues enregistrent environ 3000 séismes, dont certains très puissants, même si peu d'entre eux provoquent des dommages aux habitations et aux bâtiments. Selon les géologues, plus de 200 localités entrent dans la catégorie des sites présentant un risque sismique. En 2006, l'oblast de Naryn a subi un tremblement de terre noté 7 sur l'échelle de Richter. Son épicentre se trouvait dans le village de Kachkor, où il a endommagé 7003 maisons et 50 bâtiments publics. Sept maisons ont été complètement détruites, et 1322 sérieusement endommagées. Le 8 janvier 2007, Batken a souffert d'un séisme plus violent encore, noté 8 sur l'échelle de Richter, qui a endommagé 7137 maisons, dont 90 gravement.

\section{Séismes, inondations et glissements de terrain}

Plus de la moitié du territoire de la République est sujette aux risques d'avalanche. La saison des avalanches dure entre cinq et sept mois. Chaque année, elles provoquent de nombreuses situations d'urgence sur les routes de montagne. Plus de 10 bassins versants et 800 zones classées à risque d'avalanches ont été identifiés dans le pays. Cinquante personnes ont trouvé la mort dans les avalanches sur la période 2002-2007. 
$\mathrm{Du}$ fait d'une fonte intensive des glaciers et d'une abondance de pluies et de précipitations neigeuses, de nombreuses régions du pays subissent des inondations à chaque printemps. Il y a 3100 bassins inondables sur le territoire du Kirghizistan. En outre, 200 de ses 2000 lacs alpins ont le potentiel de provoquer des inondations. En 2003, 38 habitants du village de Sogot, dans la région d'Uzgen, ont été tués par une inondation. En 2004, aux alentours du village de Kaynama Budalyk (région d'Alay), une inondation a emporté 33 personnes, dont 17 enfants. En avril de la même année, dans la région de Kara-Suu, une ville de l'oblast d'Och, une inondation d'un volume de $60000 \mathrm{~m}^{3}$ a tué cinq écoliers. Fin mars 2005, une inondation sur le territoire du village de Sarybulak (région de Karakuldja) a détruit la route, emportant du même coup $700 \mathrm{~m}$ de lignes électriques et $1,5 \mathrm{~km}$ de lignes de communication. En 2003, après l'inondation, 50 familles de ce même village avaient été relogées.

Les glissements de terrain sont une autre catastrophe fréquente au Kirghizistan. 88 personnes y ont trouvé la mort sur la période 2002-2007. Actuellement, il y a plus de 5000 glissements de terrain actifs au Kirghizistan. Plus de 500 villages en sont virtuellement ou réellement affectés, et plus de 10000 familles vivent dans des maisons en péril. Ces inondations et ces glissements de terrain sont aussi des résultats de l'activité humaine. La principale raison de leur augmentation ces dernières années est l'érosion du sol sur les pentes montagneuses, provoquée par le surpâturage du bétail.

\section{L'anthropogenèse des risques environnementaux}

L'extraction de l'uranium, de métaux lourds et du mercure, ainsi que l'accumulation de leurs déchets comptent parmi les principales catastrophes naturelles suscitées par l'homme au Kirghizistan. Elle provoque une pollution environnementale et constitue un danger sanitaire réel et potentiel. Ces menaces sont encore aggravées par le fait que la République kirghize est située sur la section supérieure des bassins versants de la région. En conséquence, les substances contaminées issues de ce territoire peuvent atteindre les autres régions d'Asie centrale par le biais des nombreuses inondations. Le Kirghizistan compte cinq sites principaux d'exploitation : Mailuu-Suu, Kara-Balta, Ming-Kush, Kaji-Say et Ak-Tuz.

L'extraction de l'uranium a commencé ici dans les années quarante, à titre d'élément du programme nucléaire soviétique et s'est poursuivie jusqu'en 1968. Ensuite, les puits ont été fermés, mais les décharges où étaient stockés les déchets sont restées. Le 
territoire de la République abrite plus de 250 millions $\mathrm{m}^{3}$ de déchets, répartis sur 92 sites autrefois consacrés à l'industrie minière ; ils contiennent tous des radionucléides dangereux et toxiques pour l'homme. Après la faillite des compagnies minières soviétiques, ces 36 fosses de déchets et ces 25 puits, dont le volume total représente 15,7 millions de $\mathrm{m}^{3}$, ont été placés sous l'autorité du ministère des Situations d'urgence (MES) du Kirghizistan. Elles se répartissent ainsi : 31 fosses de déchets radioactifs $-7,2$ millions de $\mathrm{m}^{3} ; 5$ fosses de déchets toxiques $-5,2$ millions de $\mathrm{m}^{3}$; 25 puits de minerais divers à ciel ouvert dans les montagnes - 33 millions de $\mathrm{m}^{3}$. Des processus naturels potentiellement dangereux, tels que les glissements de terrain et les inondations, n'ont pas été pris en compte au cours de la construction de ces sites. La plupart des fosses de

Le territoire de la République abrite plus de 250 millions m $^{3}$ de déchets, répartis sur 92 sites autrefois consacrés à l'industrie minière ; ils contiennent tous des radionucléides dangereux et toxiques pour l'homme. déchets sont situées dans le lit de rivières qui ne cessent de s'éroder, provoquant ainsi la dispersion des substances radioactives dans le sol, dans l'atmosphère et dans les eaux souterraines. La situation est encore compliquée par les glissements de terrain et les tremblements de terre. Selon les scientifiques, il y a un réel danger de dispersion des déchets radioactifs dans les fleuves du Kirghizistan. En cas de pollution, les rivières du Kirghizistan qui appartiennent aux bassins fluviaux du Syr Darya et de l'Amu Darya pourraient rapidement répandre des déchets toxiques et radioactifs sur le territoire de l'Ouzbékistan et du Tadjikistan. Une dispersion à grande échelle provoquerait une catastrophe au niveau de toute la région, menaçant l'existence d'une grande partie de la population et de l'environnement local. Actuellement, le MES travaille à réenfouir les déchets. Il a dépensé 8,8 millions de soms entre 1999 et 2007, pour n'offrir qu'une sécurisation temporaire des fosses de déchets.

\section{Quitter le Kirghizistan ?}

Près de la moitié de la population kirghize vit sous le seuil de pauvreté $(55 \%$ des ruraux et $28,3 \%$ des citadins). Parmi ceux-ci, 16,9\% des ruraux et 6,9\% des urbains vivent dans une extrême pauvreté. À la suite des réformes économiques et politiques des années quatre-vingt-dix, la migration est devenue une question prioritaire pour la République kirghize. Le fort taux de chômage, la baisse du niveau de vie et l'absence de protection sociale sont les principaux facteurs responsables d'une migration 
spontanée à grande échelle, interne comme externe. Cette migration a connu un pic dans la période 1991-1993. Vers l'année 2000, les processus de migration se sont stabilisés et ont baissé, mais après les événements du 24 mars 2005, (la "révolution des tulipes") ces chiffres sont remontés. Environ 400000 citoyens de la République kirghize quittent le pays chaque année. La plupart s'en vont en Russie et au Kazakhstan. Ce phénomène est lié à une forte demande de main-d'ceuvre et à une offre de meilleurs salaires dans ces pays, ainsi qu'à l'extrême pauvreté du Kirghizistan, notamment dans le Sud.

Ce taux de migration aggrave les problèmes démographiques de la République kirghize, caractérisés par la chute du taux de natalité et une élévation de la mortalité - donc un abaissement de la croissance de la population dans un pays à la densité humaine déjà faible. Lintensité des processus de migration et l'abaissement de la croissance naturelle affectent le potentiel de main-d'ceuvre du Kirghizistan et renforcent une tendance au vieillissement de la population.

Outre la migration externe, il existe aussi des processus actifs de migration interne. Les flux migratoires s'effectuent pour l'essentiel du Sud appauvri vers les régions plus prospères du Nord, notamment la capitale, Bichkek. Depuis l'indépendance, la population de Bichkek a quasiment doublé sous l'influence de cet afflux d'immigrants. Les régions du Sud sont dépourvues de sources d'emploi, elles sont surpeuplées et souffrent d'une grave pénurie de terres. Cette migration intérieure sert très souvent de stade intermédiaire permettant de gagner de l'argent en vue d'une émigration en Russie. Les études actuelles sur les processus de migration au sein du Kirghizistan ont examiné les divers facteurs sources de migration : économiques, sociaux, culturels et même religieux. Toutefois, on s'est peu intéressé au rôle de la dégradation environnementale dans ces décisions d'émigrer.

\section{Les stratégies migratoires face aux risques naturels : avis d'experts}

Les experts - représentants d'organisations gouvernementales, non gouvernementales et internationales - se divisent sur le rôle des facteurs environnementaux dans les processus de migration. Certains y voient une réponse aux forts taux de pauvreté et de chômage, affirmant que les conditions environnementales avaient peu ou pas de signification. Pour d'autres, il existe un lien direct entre les risques environnementaux (naturels et technologiques) et la migration. Selon eux, facteurs économiques et facteurs environnementaux sont étroitement imbriqués, et il est aussi difficile qu'absurde de distinguer les migrants économiques des migrants environnementaux. 
La plupart des experts ont souligné que la majorité des habitants des zones à risques ne migrent pas. Si elles partent, les familles ne s'éloignent en général que d'une courte distance, phénomène qui tient à des ressources limitées, à des liens familiaux, et à la conviction que les effets des risques sont temporaires. En outre, il existe de nombreux cas où les migrants finissent par rentrer chez eux parce qu'ils n'ont pas réussi à s'adapter socialement, culturellement ou économiquement à leur nouvel environnement. Souvent, les experts expliquent aussi la décision de migrer par la situation financière de la famille. Cette relation est toutefois très complexe : pour certains experts, la migration n'est une option envisageable que pour les familles les plus riches. En effet, la migration implique un certain nombre de coûts tels que le transport, l'achat d'une nouvelle maison et l'achat de terre ou de bétail sur le nouveau site. Les plus pauvres étant incapables de supporter de telles dépenses, ils décident souvent de rester là où ils sont.

D'autres experts rétorquent toutefois que les familles possédant le plus de biens sont les moins susceptibles d'abandonner ou de vendre la maison, le bétail et la terre qu'ils possèdent. Les familles pauvres n'ont rien à perdre, et sont donc plus susceptibles de démarrer une nouvelle vie sur un nouveau site.

Outre le bien-être matériel de la famille, l'association entre migration et risques environnementaux varie selon son type d'habitat. Les experts soulignent ainsi que parmi toutes les zones à haut risque, le taux d'émigration est en général plus élevé 
dans les petites villes postindustrielles et les zones rurales du Kirghizistan. Cela peut être attribué aux faibles opportunités d'emplois et aux possibilités limitées de revenus, avec pour conséquence une faible infrastructure sociale et culturelle de la communauté.

Les décisions de migration dépendent aussi fortement de la solidité des liens communautaires. Ainsi, les gens sont plus susceptibles de rester, ou de ne migrer que de façon temporaire, si leur parentèle réside dans la même région. En outre, ceux qui décident de migrer partent en général dans des endroits où ils ont des parents et/ou des amis. Dans un pays où l'aide gouvernementale est limitée et rare, les réseaux sociaux sont d'une importance vitale. En conséquence, la présence de parents et/ou d'amis peut faciliter le processus d'adaptation culturelle et sociale dans un nouvel environnement, et être une source potentielle de soutien psychologique et financier.

\section{Les restrictions du choix des migrants}

Dans certains cas, seuls quelques membres de la famille migrent, les autres restant sur place. La décision de savoir qui doit rester et qui doit partir relève des stratégies migratoires familiales liées aux risques naturels. De nombreux experts soulignent que ceux qui partent sont en général des jeunes ou des gens d'âge moyen, dotés d'un niveau d'éducation plus élevé. La décision de partir s’appuie sur le présupposé que ces membres de la famille auront de meilleures opportunités d'emploi sur le nouveau site.

Les résultats de la recherche indiquent aussi que l'absence d'aide gouvernementale restreint les choix de résidence de nombreux habitants des régions à risques. La plupart des experts ont mentionné qu'ils n'avaient aucune connaissance d'un quelconque programme centralisé sur la migration environnementale. En outre, le taux d'émigration est plus bas dans les communautés où il a été procédé à une préparation aux désastres naturels et/ou des programmes d'assistance ont été mis en ceuvre.

De nombreux résidents des régions à risques ne migrent pas parce qu'ils n'ont pas le choix. La plupart des terres du Kirghizistan sont privatisées. Le manque de terres est particulièrement manifeste dans les régions du Sud où survient la majorité des catastrophes naturelles. Ceux qui souhaitent se réinstaller se voient offrir une terre de "moindre qualité", ou une terre située dans une autre zone à risques. Beaucoup de gens refusent d'aller se réinstaller ailleurs et signent un document affirmant qu'ils ont connaissance du risque potentiel, confirmant leur décision informée de rester sur des sites classés dangereux. 
Enfin, les gens refusent de partir du fait d'un attachement émotionnel à leur village, phénomène surtout apparent chez les habitants les plus âgés. Ils disent qu'ils ne peuvent pas quitter ces lieux où ils sont nés et où ils ont été élevés, et qu'ils voudraient être enterrés au même endroit que leurs ancêtres.

Si les experts ont des perceptions différentes du rôle de l'environnement dans les décisions de migration, ils s'accordent à penser que l'influence des risques environnementaux sur les divers aspects de la vie humaine va s'intensifier, et qu'en conséquence, le nombre de migrants environnementaux va s'accroître. De nombreux experts ont donc suggéré d'élaborer un programme stratégique abordant les conséquences à court et long terme des risques environnementaux sur le bien-être humain.

\section{Mailuu-Suu : survivre au milieu des déchets d'uranium}

L'équipe de recherche a travaillé dans sept territoires/sites du nord et du sud du Kirghizistan, des zones affectées par la radiation des mines d'uranium : Min-Kush et Chaek (province de Naryn), Mailuu-Suu (province de Jalal-Abad), Kara-Sogot, Nichke-Sai, Kara-Kulja, Kurshab et Manas Aiyly (province d'Och).

Mailuu-Suu est une ville de la province de Jalal-Abad. Ce fut longtemps une ville d'accès restreint, non seulement pour les étrangers mais pour les citoyens soviétiques. Située à environ 150 kilomètres d'Och, elle avait été classée "ville secrète" sous le nom de code "Boîte aux lettres 200". Mailuu-Suu est considérée comme la zone écologiquement la plus sinistrée de toute l'Asie centrale. L'Université américaine de Blacksmith, qui effectue des recherches sur les questions environnementales, a placé Mailuu-Suluu parmi les 30 villes les plus polluées du monde. Il y a deux millions de tonnes de déchets d'uranium stockées dans ses 23 décharges, qui couvrent au total $432000 \mathrm{~m}^{2}$. La radioactivité combinée de toutes les décharges de Mailuu-Suu s'élève à 5000 Curie environ. Au cours des vingt-deux ans (1946-1967) de fonctionnement de ses deux usines hydrométallurgiques, 10000 tonnes d'oxyde et de protoxyde d'uranium ont été extraites. Mailuu-Suu a survécu à la fermeture et à la réaffectation des mines d'uranium. Par la suite, une usine d'ampoules électriques a été installée dans la ville.

Si les décharges se dégradent, provoquant le glissement des déchets d'uranium dans les eaux de la rivière Mailuu-Suu, cela entraînera une catastrophe écologique régionale et empoisonnera une grande partie de la vallée de la Ferghana. Actuellement, le ministère des Situations d'urgence utilise l'argent des donateurs internationaux pour réenfouir les déchets sur le même territoire. 
L'équipe de recherche a passé une journée dans le village de Sary-Be, qui est un faubourg de Mailuu-Suu. Sary-Be est entouré de 17 décharges. Ce qu’a vu l'équipe ressemble davantage à une scène de guerre ou à un film d'horreur. Quelques bâtiments de quatre étages encore debout, mais vides, étaient entourés des ruines de maisons désossées pour récupérer des matériaux de construction. La majorité de la population était constituée de personnes âgées, la plupart des jeunes étant partis. Beaucoup de ceux qui restent sont alcooliques. À en croire un homme assez éméché, "si vous prenez 100 grammes de vodka par jour, cela réduit les effets des radiations". Mais il nous a paru surtout que l'alcool était pour lui le moyen d'oublier la terrifiante réalité de la lente destruction de son corps par les radiations, cent fois plus élevées ici que la norme autorisée selon les autorités locales et les médecins. Les entretiens avec les habitants et un médecin de la clinique locale ont révélé l'ampleur de leur impact sur la santé. Une jeune mère de quatre enfants nous a expliqué que sa mère était morte du cancer, que son père était aveugle et en phase terminale, qu'elle-même était atteinte du cancer et que ses quatre enfants avaient des goitres. Le médecin a mentionné des dizaines de maladies courantes dans leur village : cancers de tous types, perte d'énergie, problèmes de fertilité chez les femmes et d'impuissance chez les hommes, problèmes de santé mentale chez les garçons, et ainsi de suite. Les gens meurent à petit feu, et pourtant ils restent. Pourquoi ? 
Il semble qu'ils n'aient tout simplement aucun moyen de s'échapper. Ils ne bénéficient d'aucune aide du gouvernement et n'ont pas de fonds personnels pour migrer. Au bout du compte, en dépit des radiations, ils ont ici un peu de terre où faire pousser des légumes et des fruits, et de petites prairies où faire paître leur bétail dans les collines. Les membres de l'équipe ont quitté le village dans un grand désarroi, et ils ont souffert de troubles divers pendant une semaine après la visite, soit parce qu'ils avaient touché des objets sur le site, soit du simple fait d'avoir été exposés quelques heures durant à une forte irradiation.

\section{Sary-Tash : une nouvelle impasse pour les migrants}

L'équipe de recherche a étudié plusieurs autres villages situés le long du fleuve KaraKulja, un secteur à risque pour les glissements de terrain et les inondations. Il y a déjà eu deux ou trois glissements de terrain majeurs dans cette zone depuis les années quatre-vingt-dix. Un total de 209 personnes ont été déplacées. En général, le gouvernement et les autorités locales surveillent les glissements de terrain et tentent de déplacer les gens vers des endroits plus sûrs en cas de danger potentiel. Les autorités interdisent aux habitants d'utiliser des lieux dangereux et bannissent la pâture du bétail sur ces sites. Malgré le danger, certains ne partent jamais. D’autres se déplacent pour un court moment mais ne tardent pas à revenir, surtout parce que la nouvelle terre qu'on leur a donnée est trop petite et dépourvue d'eau, alors qu'ils ont une abondance de terre, d'eau et d'herbe sur leur site d'origine. De nombreuses familles quittent temporairement leurs maisons au cours des saisons dangereuses comme le printemps, ou lors de pluies abondantes, pour y revenir ensuite.

Sary-Tash (Pierre jaune) est un village de réinstallation permanente pour les personnes menacées par des glissements de terrain. Toutes les maisons ici sont neuves, mais beaucoup sont vides. Le village est bordé de collines d'un côté, et de la rive très abrupte du fleuve Kara-Kulja de l'autre. Le gouvernement a fourni des prêts à long terme qui ont couvert l'essentiel des coûts de construction des nouvelles maisons. Il a également fourni certains matériaux de construction. Les villageois ont été déplacés pour échapper à un désastre écologique. Ils font désormais face à deux autres : le manque d'eau et un nouveau glissement de terrain en préparation. Lors de leur réinstallation, les autorités leur avaient assuré que le nouveau site était sûr. Mais une fois leurs maisons bâties, quand ils ont commencé à s'installer, un nouveau glissement de terrain s'est formé sur les collines avoisinantes. Ce glissement avance chaque année, mais le gouvernement refuse d'aborder cette question, en soutenant que les gens déplacés ont 
déjà reçu leur argent et que ce glissement de terrain ne se produira pas. Le manque d'eau n'est pas considéré comme une raison suffisante pour les déplacer à nouveau. Sary-Tash n'a pas d'école et quasiment pas de services. Ses habitants n'ont pas accès à de l'eau potable ou propre pour l'irrigation. C'est un site aride, poussiéreux et à peu près vide. Les villageois se plaignent que les gens du cru les empêchent de faire paître leur bétail sur les pâturages locaux ; c'est pourquoi ils doivent les conduire sur leurs propres pâturages près de leurs sites d'origine, loin de leur lieu actuel de résidence. Les migrants de Sary-Tash vivent dans des conditions misérables, sans accès à quoi que ce soit. Ils sont coupés de leurs parents et de leur communauté, et leurs besoins fondamentaux ne sont pas satisfaits. Ce nouveau village connaît déjà un phénomène d'émigration, à mesure que les habitants comprennent qu'ils n'ont ici aucun avenir. En conséquence, ils abandonnent même les maisons neuves en quête d'un endroit plus propre à la vie. Le cas de Sary-Tash montre comment une mauvaise planification de la réinstallation, ainsi qu'une absence de terres appropriées, laisse souvent les migrants dans des conditions pires que celles qu'ils ont quittées.

\section{La chaîne de la migration environnementale}

L'équipe de recherche a étudié 30 familles migrantes vivant dans onze villages situés dans trois provinces du Kirghizistan. Dans les régions montagneuses du Kirghizistan, l'élevage est la source principale de revenu et de survie pour la population. Dans la difficile période économique qui a suivi l'effondrement de l'Union soviétique, les gens ont tenté d'accroître leur quantité de bétail. En l'absence de toute régulation étatique, ce fait a entraîné le surpâturage des terres. Ce surpâturage a provoqué une érosion des sols et des risques accrus de glissement de terrain et d'inondation. Les risques environnementaux sont devenus les principaux motifs de la migration. Aucun autre facteur (économique, social ou politique) n'a pesé aussi lourd. Le gouvernement a poussé à la réinstallation, tant par la force que par des prêts à long terme pour la construction de maisons. Il a aussi fourni de nouvelles terres aux habitants. Toutefois, du fait de la surpopulation du sud du Kirghizistan, les seules terres qui leur ont été offertes étaient de dimensions très restreintes et situées dans des endroits quasiment dépourvus d'eau, sans pâturages à proximité. Les migrants sont donc passés à des sites impropres à l'élevage et à l'agriculture. Ils ont dépensé leurs économies et vendu leur bétail pour migrer, avant de s'apercevoir qu'ils pouvaient à peine survivre sur leur nouveau site d'installation. Au cours du processus de migration, ils ont réussi à préserver certains liens familiaux, mais ils ont perdu l'essentiel de leurs réseaux sociaux. 
Nous trouvons donc au début de la chaîne de migration des réalités économiques difficiles et une absence de contrôle des activités humaines comme principale raison de la dégradation environnementale, qui contraint les gens à émigrer. Au bout de la même chaîne, nous trouvons des familles migrantes disloquées qui ont perdu leur environnement naturel, social et culturel, ainsi que la plupart de leurs opportunités économiques.

\section{Pour ceux qui restent, une vie dans les ruines}

Notre recherche a aussi étudié 36 familles non migrantes vivant dans sept villages de trois provinces du Kirghizistan. Les résultats montrent que ceux qui sont restés en arrière s'en sortent en général mieux économiquement que ceux qui sont partis. Ils préservent leurs terres et leur bétail. Toutefois, ils sont massivement affectés par la détérioration de l'environnement et par l'émigration. Les principaux effets des problèmes environnementaux se font sentir sur leur santé physique et psychologique (surtout dans les sites des anciennes mines d'uranium) et sur leur niveau de vie (déclin des récoltes et perte de bétail). Le résultat de l'émigration est la perte du milieu 
socioculturel, une population vieillissante, une jeunesse démoralisée et une détérioration des infrastructures. Certains villages peuvent perdre jusqu'à 80 familles en une seule année. Pour ceux qui restent, la pression s'accumule : la plupart veulent partir à cause des problèmes d'environnement, mais ils n'en ont pas la possibilité. D'une part, ils redoutent les difficultés économiques sur les nouveaux sites, et, d'autre part, ils n'ont pas les moyens de partir. En conséquence, ils continuent à se réveiller tous les jours dans l'horrible réalité de leur corps détruit par chaque seconde de vie dans ces zones radioactives, sous la menace permanente d'un glissement de terrain qui pourrait emporter leur maison.

\section{Conclusion : un scénario perdant-perdant}

Le cas kirghize montre que les processus de migration au Kirghizistan ont une forte coloration environnementale et que les problèmes environnementaux provoquant la migration et le déplacement de vastes groupes de population n'ont que des impacts négatifs sur la vie des communautés locales. Ce qui caractérise la migration environnementale, c'est qu'en général, il ne s'agit pas d'un choix, mais de l'unique solution dans un scénario où toutes les issues sont perdantes, tant pour ceux qui migrent que pour ceux qui restent. Leurs réseaux sociaux, bâtis sur une vie entière, sont détruits. Leurs stratégies de subsistance, héritées de nombreuses générations, perdent tout leur sens. Leur culture, fortement liée à l'identité et à l'unité tribale, disparaît.

Comme l'a révélé cette étude, quasiment toutes les catastrophes ont des racines anthropogéniques, qu'il s'agisse de la grande industrie gouvernementale ou de pratiques individuelles d'élevage à petite échelle. Cependant, le gouvernement actuel du Kirghizistan s'intéresse surtout aux conséquences sans aborder les causes réelles. Des initiatives à tous les niveaux sont nécessaires pour préserver les collines, les forêts, les lacs et les fleuves des écosystèmes kirghizes. Une solution peut être la reprise des méthodes autochtones traditionnelles d'élevage et d'agriculture. Les nomades kirghizes ont toujours veillé à traiter les pâturages avec grand soin. Ils n'étaient pas attachés à un endroit, mais se déplaçaient en fonction des aléas du climat, s'inscrivant ainsi dans un écosystème complexe et interconnecté. Beaucoup de ces pratiques et une grande part du savoir écologique traditionnel ont été perdus au cours du siècle dernier, marqué par l'exploitation collective des terres et la sédentarisation. Il est également important de reprendre l'élevage des races autochtones de moutons et la culture des variétés de fruits et de légumes propres au Kirghizistan, et bien 
mieux adaptés aux conditions environnementales locales. Par exemple, le mouton autochtone de la vallée de l'Alay consomme beaucoup moins d'herbe et exerce une pression nettement moins forte sur les pâturages que quantité de nouvelles espèces hybrides introduites au Kirghizistan au cours de la période soviétique. Un autre exemple, l'abricot Kasek, qui n'a besoin d'un arrosage que deux à trois fois par an, contrairement à de nombreux abricotiers importés, qui exigent six à sept fois plus d'eau. Il est particulièrement adapté à la province méridionale de Batken, fortement affectée par les sécheresses. Plusieurs initiatives sont actuellement en cours au Kirghizistan, visant à préserver l'agro-biodiversité de la région à l'aide de méthodes autochtones. Retrouver la sagesse et les pratiques traditionnelles pourrait aider de nombreuses communautés d'Asie centrale à restaurer l'équilibre écologique perdu, premier pas vers la restauration du milieu social et culturel des communautés kirghizes exposées à la migration forcée.

Article traduit par Françoise Bouillot

\section{Bibliographie}

- I. Stepanenko, Природно-климатические условия Кыргызстана, ЦАИК, 1997.

- M. Radjabov, Проблемы региональной безопасности в Центральной Азии на пороге XXI века, Газета "Навигатор" (Казахстан), 2008.

- I. D. Zvyagelskaya, K вопросу об угрозах в Центральной Азии, Материалы научнопрактического семинара, “10 лет СНГ: некоторые итоги”, СЕРИЯ: Научные доклады, n5, Июль 2001.

- Y. Gruzdov, Где хвост уранового дракона? Кыргызстан, n²11, за март 2003

- C. Mamajakyp Uulu, E. B. Kamcgybeov, C. M. Magazova, D. Nazarbekov, A. K. Nartaeva, S. M. Mamatov, Изменение клеток периферической крови у жителей, проживающих в районе захоронения радиоактивных отходов, Научно-исследовательский отдел ДМСРЦ Министерства здравоохранения Кыргызской Республики, Кыргызско-Российский Славянский Университет (г. Бишкек), 2005.

- A. I. Tishin, Процессы социальной диссипациив Кыргызской Республике, Кыргызский Национальный университет, г. Бишкек, 2007. 